\title{
Life-saving vascular access after combined liver and kidney transplantation: A challenging access to the right atrium
}

The Journal of Vascular Access

$1-4$

(C) The Author(s) 2019

Article reuse guidelines:

sagepub.com/journals-permissions DOI: $10.1177 / 1129729818820516$

journals.sagepub.com/home/jva

(S)AGE

\author{
Chiara Grimaldi', Alessandro Crocoli' ${ }^{2}$ Roberta Angelico', \\ Maria Cristina Saffioti', Simona Gerocarni Nappo ${ }^{3}$, \\ Fabrizio Chiusolo 4 , Francesca Tortora ${ }^{4}$, \\ Sergio Giuseppe Picardo ${ }^{4}$, Luca Dello Strologo ${ }^{5}$, \\ Maria Sole Basso ${ }^{6}$, Lidia Monti ${ }^{7}$, Paolo Guccione ${ }^{8}$, \\ Massimo Rollo ${ }^{7}$ and Marco Spada!
}

\begin{abstract}
Exhaustion of vascular accesses is a major complication in patients undergoing hemodialysis, especially in pediatric setting. We report the case of a boy treated for loss of hemodialysis access after a combined liver-kidney transplantation and transient renal dysfunction. An interventional dilatation of calcific superior vena cava allowed to insert a stable central venous line for dialysis until full graft recovery. Careful management of central lines allows to spare the main vessels and reduces the need for unusual accesses.
\end{abstract}

\section{Keywords}

End-stage liver disease, end-stage kidney disease, liver transplantation, kidney transplantation, children, hemodialysis

Date received: 24 September 2018; accepted: 20 November 2018

\section{Introduction}

Solid organ transplantation for end-stage diseases is a standard of care for a variety of conditions in pediatric population; management of these patients may be challenging in both pre- and post-transplantation period. ${ }^{1,2}$ One of the most common issues, especially for end-stage kidney disease, is the preservation of reliable vascular access over the course of disease, also considering the long life expectancy in children and the likely need of more than one transplant during their life span. In this respect, knowledge and skills to manage patients with exhaustion of vascular access is a necessary prerequisite in order to avoid life-threatening complications.

Even if artero-venous fistula is the gold standard for hemodialysis, ${ }^{3,4}$ this access may be challenging for both technical and anatomical reasons in younger children. ${ }^{5}$ Patients with end-stage kidney disease may face repeated catheter placements with progressive loss of conventional vascular access sites. ${ }^{6}$
'Department of Pediatric Surgery and Transplantation, Bambino Gesù Children's Hospital, IRCCS, Rome, Italy

2Department of Pediatric Surgery, Bambino Gesù Children's Hospital, IRCCS, Rome, Italy

3Pediatric Urology Unit, Bambino Gesù Children's Hospital, IRCCS, Rome, Italy

${ }^{4}$ Department of Anesthesia and Critical Care, ARCO, Bambino Gesù Children's Hospital, IRCCS, Rome, Italy

${ }^{5}$ Transplant Clinic, Department of Pediatric Specialties, Bambino Gesù Children's Hospital, IRCCS, Rome, Italy

'Unit of Gastroenterology, Hepatology and Nutrition, Bambino Gesù Children's Hospital, IRCCS, Rome, Italy

${ }^{7}$ Radiology Department, Bambino Gesù Children's Hospital, IRCCS, Rome, Italy

${ }^{8}$ Department of Pediatric Cardiology and Cardiac Surgery, Bambino Gesù Children's Hospital, IRCCS, Rome, Italy

Corresponding author:

Alessandro Crocoli, Department of Pediatric Surgery, Bambino Gesù Children's Hospital, IRCCS, Piazza Sant'Onofrio 4, 00165 Rome, Italy. Email: alessandro.crocoli@opbg.net 


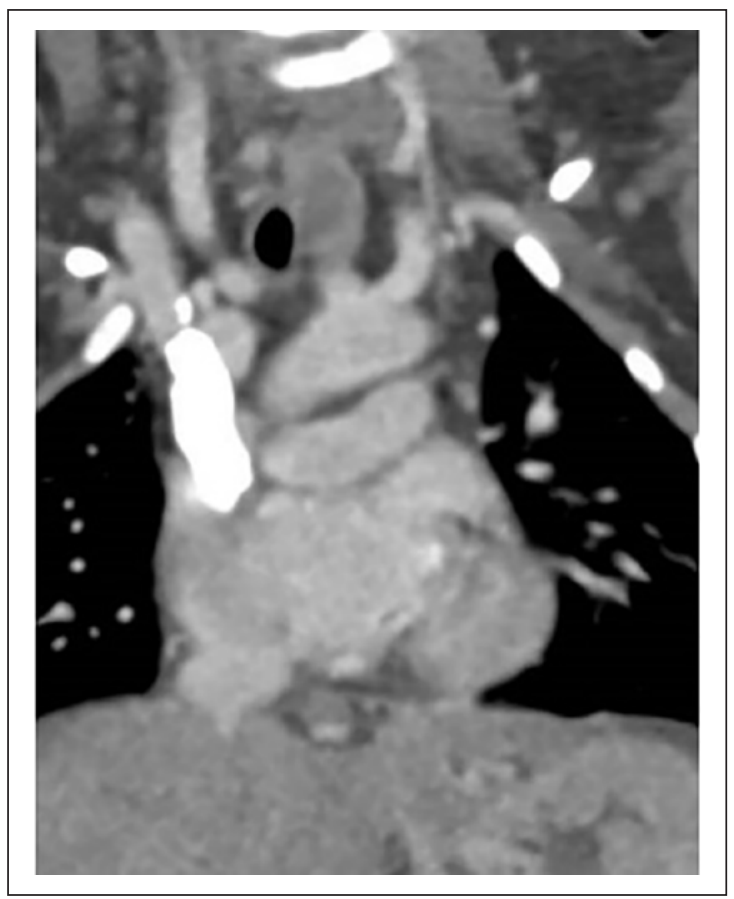

Figure I. Superior vena cava stenosis secondary to intraluminal calcium apposition.

Taking into account actual organ shortage and long waiting list time for children, ${ }^{2}$ additional attention should be put in order to further reduce catheter-related complications. Moreover, effective vascular access may be crucial also in the management of post-transplant complications such as primary kidney dysfunction. In the setting of challenging transplant procedures, vascular exhaustion can become a major issue in the immediate post-transplant period, hence jeopardizing the outcome, in terms of both graft and patient survival. ${ }^{7}$

Therefore, long-term dependency on catheter-based dialysis may require unconventional approaches to perform life-saving procedures.

\section{Case report}

A 9-year-old boy with nephronophthisis and liver fibrosis was assessed for a combined liver and kidney transplant. Pre-transplant vascular mapping showed a diffuse thrombosis of the major vessels including right internal and common iliac veins and right internal jugular vein. Moreover, the superior vena cava appeared significantly narrowed by a concentric calcium apposition (Figure 1). Functional evaluation of caval circulation was performed by direct measurements of pressures in the inferior vena cava, superior vena cava, and in the right atrium, showing no pressure gradient.

At the time of the combined liver and kidney transplantation, two different central venous catheters (CVCs) were placed, one for hemodialysis and one for the administration of emergency medications. Both lines were placed in the left internal jugular vein with the tips upstream of the superior vena cava stenosis. Both catheters were successfully used during and immediately after transplantation. The patient showed immediate good liver function while a delayed graft function occurred for the kidney due to acute tubular necrosis, leading to the need of post-transplant dialysis.

After few days, a dialysis catheter malfunction secondary to device dislodgment occurred, thus requiring a more stable access to administer hemodialysis until full kidney graft function recovery on post-transplant day 37 .

\section{Technical keypoints}

As a consequence of a poorly controlled hyperparathyroidism, diffuse calcifications of both arterial and venous major vessels were recorded at pre-transplant evaluation. In particular, a circumferential calcification of the superior vena cava with significant narrowing of the internal lumen was detected. Nevertheless, direct measurement of intravascular pressures confirmed a normal flow to the right atrium; hence, those findings were not considered a contraindication to transplantation.

Given the need for caval clamping during liver transplantation, the patient had no access through the iliac axis. Moreover, in this specific case, the iliac venous axis was not approached since the right side was thrombosed and the left one had to be spared for the implantation of the kidney graft.

In the post-transplant period, loss of vascular access was due to the dislodgment of multiple central lines due to high flow/high pressure dialysis against a severe superior vena cava stenosis.

\section{Procedure}

Access via the left jugular vein;

Direct confirmation of a severe stenosis of the superior vena cava with diffuse and concentric calcification with an internal diameter of $3 \mathrm{~mm}$ (Figure 2(a));

The stenotic superior vena cava is dilated from 3 to $7.5 \mathrm{~mm}$ (pressure of dilatation balloon: 14 atmospheres). Figure 2(b) and (c) shows the intravascular balloon before and after dilatation;

Placement of 8-Fr dialysis catheter through the stenosis with the tip in the right atrium (Figure 2(d)). A second $3-\mathrm{F}$ peripherally inserted central catheter (PICC) is placed via the right basilic vein. The catheter tip is confirmed to be in the right atrium. 


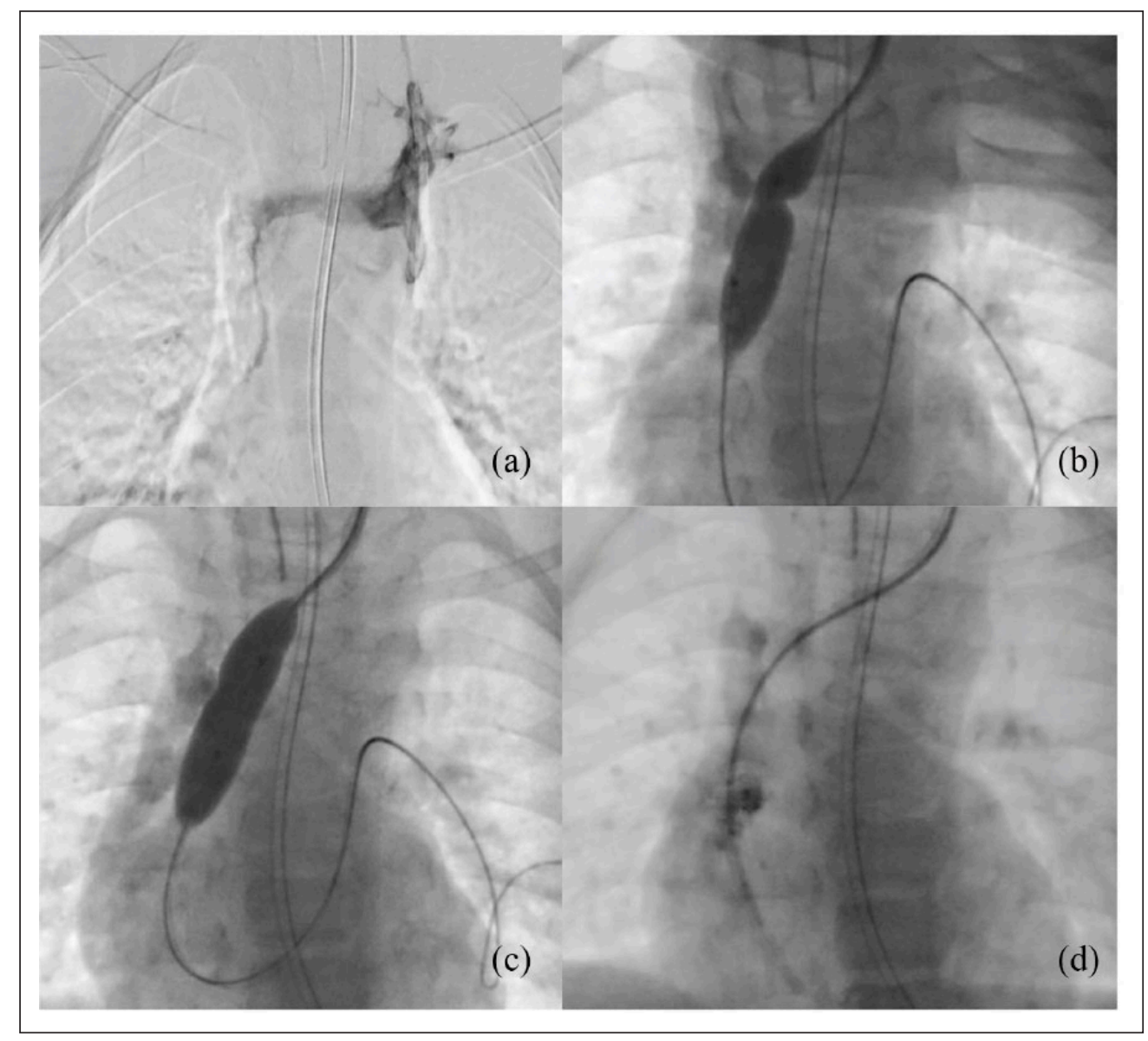

Figure 2. (a) Angiographic image of superior vena cava stenosis, (b and c) angioplastic procedure: pressure dilatation of stenotic vena cava and (d) central venous catheter placed in previously-stenotic superior vena cava.

\section{Comments and conclusion}

Exhaustion of hemodialysis access is a well-known issue in management of children waiting for renal transplantation. We discuss here a patient for whom challenging vascular access became a major issue after a combined liver and kidney transplantation, putting both patient and graft survival at risk.

We report an unconventional approach to the right atrium that allowed establishing a safe and long-term hemodialysis access.

As overall survival improves, patients undergo cycles of vascular access failure, temporary catherization, and revision/replacement of central lines, progressively exhausting all standard access sites. ${ }^{8}$ All efforts should be put in preventing exhaustion of conventional vascular access: still, a resourceful multidisciplinary team should be ready to overcome challenging situations to approach and solve specific and life-threatening issues. ${ }^{9}$

\section{Declaration of conflicting interests}

The author(s) declared no potential conflicts of interest with respect to the research, authorship, and/or publication of this article.

\section{Funding}

The author(s) received no financial support for the research, authorship, and/or publication of this article.

\section{References}

1. Jimenez-Rivera C, Nightingale S, Benchimol EI, et al. Outcomes in infants listed for liver transplantation: a retrospective cohort study using the United Network for Organ Sharing database. Pediatr Transplant 2016; 20(7): 904-911.

2. 2010 SRTR/OPTN annual report: chapter index: data tables 2018, https://srtr.transplant.hrsa.gov/annual_reports/2010 /chapter_index.htm

3. Rehman R, Schmidt RJ and Moss AH. Ethical and legal obligation to avoid long-term tunneled catheter access. Clin J Am Soc Nephrol 2009; 4(2): 456-460.

4. Baracco R, Mattoo T, Jain A, et al. Reducing central venous catheters in chronic hemodialysis - a commitment to arteriovenous fistula creation in children. Pediatr Nephrol 2014; 29(10): 2013-2020.

5. Souza RA, Oliveira EA, Silva JM, et al. Vascular access in children. J Bras Nefrol 2011; 33(4): 422-430.

6. Rahman S and Kuban JD. Dialysis catheter placement in patients with exhausted access. Tech Vasc Interv Radiol 2017; 20(1): 65-74. 
7. Dharnidharka VR, Fiorina $\mathrm{P}$ and Harmon WE. Kidney transplantation in children. $N$ Engl J Med 2014; 371(6): 549-558.

8. Funaki B, Zaleski GX, Leef JA, et al. Radiologic placement of tunneled hemodialysis catheters in occluded neck, chest, or small thyrocervical collateral veins in central venous occlusion. Radiology 2001; 218(2): 471-476.

9. Shroff R, Sterenborg RB, Kuchta A, et al. A dedicated vascular access clinic for children on haemodialysis: two years' experience. Pediatr Nephrol 2016; 31(12): 2337-2344. 